\title{
NMDA receptors are expressed in human ovarian cancer tissues and human ovarian cancer cell lines
}

This article was published in the following Dove Press journal:

Clinical Pharmacology: Advances and Applications

23 October 2015

Number of times this article has been viewed

\section{William G North \\ Fuli Liu \\ Ruiyang Tian \\ Hamza Abbasi \\ Bonnie Akerman}

Department of Physiology and Neurobiology, Geisel School of Medicine at Dartmouth College, Lebanon, $\mathrm{NH}$, USA
Correspondence: William G North Department of Physiology and Neurobiology, Geisel School of Medicine at Dartmouth College, I Medical Center Drive, Lebanon, NH 03756, USA

Tel +l 6036507736

$\mathrm{Fax}+16036506130$

Email william.g.north@dartmouth.edu
Abstract: We have earlier demonstrated that breast cancer and small-cell lung cancer express functional NMDA receptors that can be targeted to promote cancer cell death. Human ovarian cancer tissues and human ovarian cancer cell lines (SKOV3, A2008, and A2780) have now been shown to also express NMDA-receptor subunit 1 (GluN1) and subunit 2B (GluN2B). Seventeen ovarian cancers in two arrays were screened by immunohistochemistry using polyclonal antibodies that recognize an extracellular moiety on GluN1 and on GluN2B. These specimens comprised malignant tissue with pathology diagnoses of serous papillary cystadenocarcinoma, endometrioid adenocarcinoma, and clear-cell carcinoma. Additionally, archival tissues defined as ovarian adenocarcinoma from ten patients treated at this institute were also evaluated. All of the cancerous tissues demonstrated positive staining patterns with the NMDA-receptor antibodies, while no staining was found for tumor-adjacent normal tissues or sections of normal ovarian tissue. Human ovarian adenocarcinoma cell lines (A2008, A2780, SKOV3) were demonstrated to express GluN1 by Western blotting, but displayed different levels of expression. Through immunocytochemistry utilizing GluN1 antibodies and imaging using a confocal microscope, we were able to demonstrate that GluN1 protein is expressed on the surface of these cells. In addition to these findings, GluN2B protein was demonstrated to be expressed using polyclonal antibodies against this protein. Treatment of all ovarian cell lines with antibodies against GluN1 was found to result in decreased cell viability $(P<0.001)$, with decreases to $10 \%-25 \%$ that of untreated cells. Treatment of control HEK293 cells with various dilutions of GluN1 antibodies had no effect on cell viability. The GluN1 antagonist MK-801 (dizocilpine maleate) and the GluN2B antagonist ifenprodil, like antibodies, dramatically decreased the viability of A2780 ovarian tumor cells $(P<0.01)$. Treatment of A2780 tumor xenografts with ifenprodil (2.5 mg/kg body weight/day) significantly reduced tumor growth in nu/nu mice. Our findings suggest that both GluN1 and GluN2B proteins as membrane components could be readily available targets for the treatment of most ovarian cancers.

Keywords: ovarian cancer, NMDA receptors, inhibitors, antibodies, potential therapy

\section{Introduction}

NMDA receptors (NMDARs) constitute important calcium channels that are chiefly found within the central nervous system. Within the central nervous system, these receptors are involved in synaptic plasticity and memory function. Overstimulation of the calcium channel results in cell death. ${ }^{1}$ Small-drug antagonists of the GluN1 protein, such as memantine and dizocilpine maleate (MK-801), have been developed and used as a form of therapy for Alzheimer's disease by irreversibly blocking the excessive calcium influx through these channels. ${ }^{2-4}$ NMDARs comprise four heterodimerized subunits forming an ion channel that is regulated by glycine and glutamate..$^{5,6}$ 
Each NMDAR subunit contains four transmembrane domains, resulting in respective extracellular ligand-binding and intracellular activating elements. The extracellular element of each protein forming the heterodimer can be potentially useful for developing new targeted treatments with antibodies. Our laboratory has shown by immunohistochemistry and Western analysis that expression of NMDARs by normal tissues is only apparent for the central nervous system, testes, and uterus. We have also demonstrated that a functional receptor is expressed by breast cancer, small-cell lung cancer, and neuroblastoma. ${ }^{7}$ Targeting this receptor with the small-molecule antagonists MK-801 and memantine significantly decreases in vitro cell viability and in vivo xenograft tumor growth. This paper describes an investigation into the expression of NMDARs by human ovarian tumors and cell lines, along with the consequences of targeting these receptors with antagonists, and antibodies against an external region of the GluN1 and GluN2B subunits of this receptor. Findings indicate NMDARs are critical for ovarian cancer cell growth and can be used as a potential target for successful cancer therapy.

\section{Materials and methods NMDAR antibodies}

We have previously described production and characteristics of rabbit polyclonal antibodies against an external region of the GluN1 protein, referred to by the acronym "PANN1". 1,8 The decapeptide representing this region used to create the antibody was MSIYSDKSIH amide. Antibodies were used as IgG preparations after precipitation from serum with 50\% ammonium sulfate solution, centrifugation at $14,000 \times g$, and reconstitution in phosphate-buffered saline (PBS). They were also used as affinity-purified preparations prepared through binding to a peptide antigen Sepharose column with acid elution followed by dialysis. Likewise, rabbit polyclonal antibodies were generated to an external and unique segment of GluN2B protein using the decapeptide YKDAHEKDDF amide, and are herein referred to as "MANDA2B".

\section{Immunohistochemistry}

Tissue expression was determined using immunohistochemistry employing the BioGenex (Fremont, CA, USA) supersensitive link-label immunohistochemistry-detection kit and PANN 1 and MANDA2B IgG antibody preparations diluted in $\mathrm{PBS} / 0.4 \mathrm{M} \mathrm{NaCl}$ containing $1 \%$ horse serum (PSHS). Ovarian cancer tissue arrays were obtained from Tissue Array Networks, specifically catalog numbers OV241 and OV242. Tissue array OV241 contained samples from seven patients with pathology diagnoses of serous papillary cystadenocarcinoma and endometrioid adenocarcinoma, along with normal tissue samples adjacent to the cancer. Tissue array OV242 contained samples from ten patients with pathology diagnoses of serous papillary cystadenocarcinoma, endometrioid adenocarcinoma, and clear-cell carcinoma, along with normal tissue samples adjacent to the cancer. As well as these arrays, ovarian cancer tissues identified as adenocarcinoma from ten patients treated at this institute were retrieved from an archival library. The immunohistochemistry protocol used was as follows: antigen recovery was performed by heating in $0.01 \mathrm{M}$ sodium citrate buffer at $80^{\circ} \mathrm{C}$ for 30 minutes. Endogenous peroxidase activity and nonspecific staining were blocked with the kit reagents. The tissues were incubated overnight with the primary MANDA antibodies. Tissues were then washed in PSHS, followed by incubation with the kit reagents Link (peroxidase-bound goat antirabbit IgG1), and diaminobenzidine, then washing in PSHS. Poststaining was carried out with hematoxylin. Mounted tissues were imaged on an Olympus (Tokyo, Japan) BX51 microscope employing a QImaging (Surrey, BC, Canada) MicroPublisher 3.3 camera.

\section{Ovarian cancer cell lines and cell culture}

Ovarian cancer cell lines SKOV3, A2780, and A2008 were grown in Dulbecco's modified Eagle's medium/F12 media (HyClone; GE Healthcare, Little Chalfont, UK) supplemented with 10\% fetal bovine serum (HyClone) and an antibiotic solution (Sigma-Aldrich Co, St Louis, MO, USA). SKOV3 (HTB-77; American Type Culture Collection, Manassas, VA, USA), A2780 (Health Protection Agency 93112519), and A2008 (Dartmouth Medical School) are epithelial cell lines that all originated from a human ovarian adenocarcinoma. Dartmouth Medical School Institutional Review Board approved of the use of the above human cell lines.

\section{Western blotting}

Protein lysates from ovarian cancer cell lines A2780, SKOV3, and A2008 were generated in a sodium dodecyl sulfate (SDS) lysis buffer (2\% SDS, 1\% 2'-[N-cyclohexylamino]ethane sulfonic acid, 1\% glycerol) from Sigma-Aldrich. An aliquot of each lysate (2-15 $\mu$ g protein) was incubated with $4 \times$ gelloading buffer (0.1 M Trizma $\mathrm{HCl}, 10 \%$ glycerol, $2 \% \mathrm{SDS}$, $0.001 \%$ bromophenol blue) from Sigma-Aldrich, and 50 $\mathrm{mM}$ dithiothreitol at $100^{\circ} \mathrm{C}$ for 10 minutes. The proteins in samples were separated by $10 \%$ and $12 \%$ SDS PAGE at $75 \mathrm{~V}$ for 30 minutes, followed by $150 \mathrm{~V}$ for 80 minutes. Following electrophoresis, proteins were transferred to polyvinylidene difluoride Immobilon-P membranes (EMD Millipore, 
Billerica, MA, USA) at $35 \mathrm{~V}$ and $4^{\circ} \mathrm{C}$ overnight with transfer buffer comprising $250 \mathrm{mM}$ Trizma $\mathrm{HCl}, 200 \mathrm{mM}$ glycine, and $6 \%$ methanol, pH 8.3 (Sigma-Aldrich). The transfer polyvinylidene difluoride membrane was then immersed in Ponceau S total protein stain (Sigma-Aldrich) for 10 minutes, and imaged using a FluorChem 8900 imager (ProteinSimple, San Jose, CA, USA). The Ponceau S staining was used as a control for assessing the total protein content of each sample. Each membrane was then incubated in SuperBlock PBS buffer (Thermo Fisher Scientific, Waltham, MA, USA) for 1 hour at room temperature with gentle agitation, and then incubated in the same manner overnight at $4^{\circ} \mathrm{C}$ or for 60 minutes at ambient temperature with a commercial anti-NMDAR1 antibody preparation (4204; Cell Signaling Technology, Danvers, MA, USA) diluted 1:5,000 in PBS containing 5\% nonfat dry milk following the manufacturer's recommendations, or with IgG from MANDA2B antiserum. The membrane was washed with $0.01 \%$ Tween in PBS and incubated with gentle agitation with a 1:5,000 dilution of a second goat antirabbit antibody conjugated with horse radish peroxidase (7074; Cell Signaling Technology) for 60 minutes. Finally, the membrane was washed with PBS and incubated with SuperSignal WestDura Extended Duration Substrate (Thermo Fisher Scientific) and imaged using the FluorChem 8900 imager.

\section{Immunocytochemistry and confocal microscopy}

The coverslips were seeded with 10,000 SK-OV3, A2780, or A2008 cells and grown to $100 \%$ confluence. The cells were fixed with formalin (Thermo Fisher Scientific), permeabilized with $0.5 \%$ NP40 (Sigma-Aldrich), blocked with phosphate-buffered gelatin, and stained overnight at $4{ }^{\circ} \mathrm{C}$ with a 1:500 serum-equivalent dilution of an $\mathrm{IgG}$ preparation of our rabbit PANN1 antibodies. The coverslips were washed five times with $1 \%$ bovine serum albumin in PBS and incubated with a 1:2,000 $(1 \mu \mathrm{g} / \mathrm{mL})$ dilution of Alexa Fluor 488 goat antirabbit secondary antibody (Thermo Fisher Scientific) for 60 minutes. The coverslips were then washed five times with PBS containing $1 \%$ bovine serum albumin, postfixed with $4 \%$ paraformaldehyde, and mounted in VectaShield (Vector Laboratories, Burlingame, CA, USA) on glass slides. The slides were visualized with an Olympus BX61WI fluorescent confocal microscope employing a Hamamatsu Photonics KK (Hamamatsu, Japan) Orca-ER C4742-80 camera.

\section{Alamar Blue cell viability assay}

Trypsinized ovarian cancer cells (A2780, SKOV3, and A2008) were seeded at approximately 20,000 per well into a 96-well plate. The cells were allowed to recover from trypsinization for 24 hours. They were then treated with various dilutions of the GluN1 antagonist MK-801 (100-800 $\mu \mathrm{M}, \mathrm{n}=8$ ) and an $\mathrm{IgG}$ preparation of PANN1 antibodies precipitated from serum with $50 \%$ saturated $\left(\mathrm{NH}_{4}\right)_{2} \mathrm{SO}_{4}(1: 5,000,1: 2,500,1: 1,000,1: 500,1: 100,1: 50$, $1: 25$, and $1: 10$ relative to the original serum concentration, $\mathrm{n}=8$ ) for 72 hours. A2780 cells were treated with concentrations from 100 to $800 \mu \mathrm{M}$ of the GluN2B antagonist ifenprodil over 48 hours $(n=16)$. The Alamar Blue cell viability reagent was added to each well at a concentration of $10 \%$ and incubated at $37^{\circ} \mathrm{C}$ for $1-4$ hours or until the substrate had changed to a pink color. The plate was read on a Synergy HT fluorescent plate reader (BioTek Instruments Inc., Winooski, VT, USA) at a fluorescence excitation wavelength of $570 \mathrm{~nm}$ and emission wavelength of $590 \mathrm{~nm}$. The cell treatments were performed in quadruplicate, and each experiment was performed five times. Cell viability was normalized to untreated cell values and expressed as a percentage of those values.

\section{Treatment of human ovarian cancer xenografts with receptor antagonist}

Trypsinized A2780 ovarian cancer cells $\left(2 \times 10^{7}\right)$ were placed subcutaneously into the flank of 7 -week-old male nu/nu mice and allowed to develop as tumor xenografts $>5 \mathrm{~mm}$ in diameter. The growth of these tumors was measured over a 3 -day period, and then they were treated daily with vehicle or $2.5 \mathrm{mg} / \mathrm{kg}$ body weight of the GluN2B antagonist ifenprodil for 8 days. Tumor size was measured each day with a micrometer over this period of treatment. Daily measurement of animal body weight was also performed.

\section{Statistical evaluation}

Results were analyzed by analysis of variance and the Student-Newman-Keuls test. Longitudinal growth data were evaluated using repeated-measures analysis of variance. Significance was determined to be present for $P<0.05$.

\section{Results Immunohistochemistry}

All of the ovarian cancer tissue samples subjected to immunohistochemistry using the PANN1 and MANDA2B antibodies resulted in a positive staining pattern. The tissues demonstrated a membrane and cytosolic staining pattern and had various degrees $(+3$ and +4$)$ of staining intensities. Normal ovarian tissues had no positive staining. Figure 1A depicts three ovarian cancer tissues with a positive staining pattern for GluN1 and no staining of the corresponding 


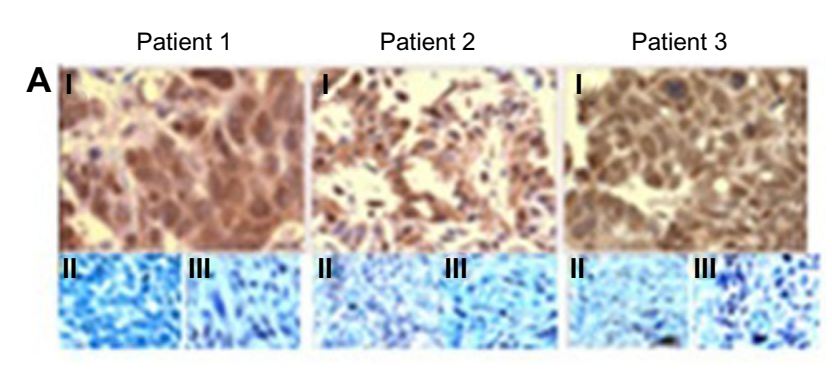

B

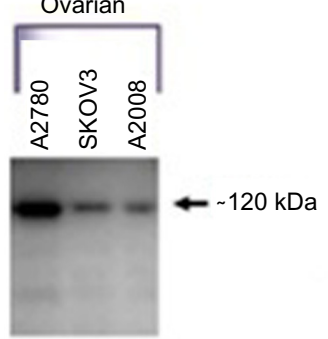

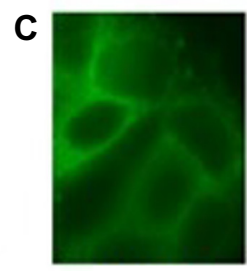

A2780

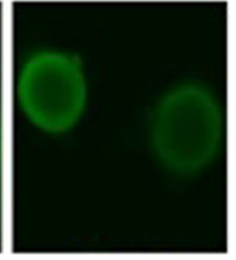

SKOV3

Figure I Antibody Staining for presence of NMDA Receptors.

Notes: (A) Immunohistochemical staining of ovarian cancers from three patients. I, Positive staining of tumors with NMDAR I antibodies; II, negative staining of normal adjacent tissue; III, negative staining with antibodies blocked by $20 \mu \mathrm{g} / \mathrm{mL}$ peptide antigen. (B) Sodium dodecyl sulfate polyacrylamide-gel electrophoresis using $12.5 \%$ gels and Western blot analysis with anti-NMDARI antibodies from Cell Signaling Technology. Shown is the presence of NMDARI protein at $\sim 120 \mathrm{kDa}$ in equally loaded lysates from A2780, SKOV3, and A2008 ovarian cancer cell lines. (C) Confocal imaging of A2780 and SKOV3 ovarian cancer cells with PANNI antibodies.

normal tissue adjacent to the cancerous tissue. For a negative control, tumor tissues were incubated with PANN1 antibody preparations in the presence of an excess $(20 \mu \mathrm{g} / \mathrm{mL})$ of antigen peptide. These negative controls gave no positive staining pattern demonstrating the specificity of the antibodies for the peptide segment of the receptor protein. In the same manner, specificity of staining was demonstrated for GluN2B antibodies.

\section{Protein expression in ovarian cancer cell lines}

GluN1 expression can be detected using Western blotting in all three of the ovarian cancer cell lines (SKOV3, A2780, and A2008). The cell lysates were normalized to total protein content. The three cell lines demonstrated various degrees of expression, with A2780 exhibiting the highest expression and A2008 and SKOV3 expressing similar amounts (Figure 1B). The GluN1 protein was detected as a single band located at $\sim 120 \mathrm{kDa}$. GluN2B expression was only examined for A2780 cells, and this protein was also demonstrated to be clearly present.

\section{Immunocytochemistry}

We were able to demonstrate that all three of the ovarian cancer cell lines express GluN1 on the surface of the cells. The cell lines exhibited a membrane-staining pattern with

slight detection of the GluN1 protein in the cytosol of the cell. Figure 1C are representative images of two of the cell lines, which were created by taking $10 \times 1 \mu \mathrm{m}$ confocal image slices and stacking them on top of each other.

\section{Cell viability}

Utilizing the Alamar Blue assay, we were able to demonstrate that treatment of the ovarian cancer cell lines with various dilutions of the PANN1 antibody significantly $(P<0.001)$ decreased the viability of the cells (Figure 2 ). We used the breast cancer cell line MCF7 as a positive control, since our laboratory has demonstrated in the past that PANN1 antibody killed MCF7 cells in vitro and in vivo. The SKOV3 and A2008 cell lines had very similar killing curves compared to MCF7. We were able to demonstrate a decrease in cell viability to the extent of $75 \%-90 \%$ when compared to untreated cells. The viability of HEK293 cells treated with various concentrations of PANN1 showed no change in cell viability. The effects of MK-801 were only examined on A2780 cells, and decreased viability with a $50 \%$ decrease at approximately $400 \mu \mathrm{M}(\mathrm{n}=8$ for each concentration, $P<0.01)$. The GluN2B antagonist ifenprodil likewise produced a decrease in cell viability of A2780 ovarian cancer cells (Figure 3A) in concentrations ranging from 100 to $800 \mu \mathrm{M}$, with a $50 \%$ decrease also at $\sim 400 \mu \mathrm{M}$ of antagonist ( $\mathrm{n}=16$ for each concentration, $P<0.003)$.

\section{Ovarian cancer xenografts}

The ovarian cancer cell line A2780 produced substantial tumors in all injected nu/nu mice, and those treated with

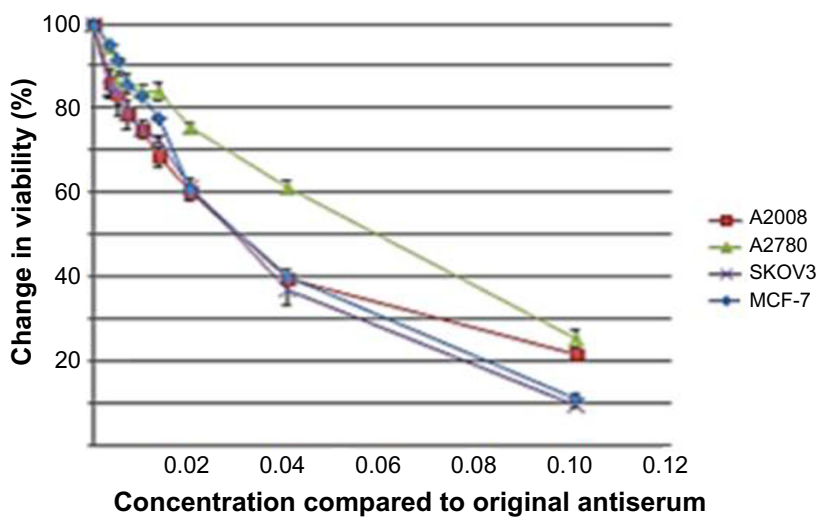

Figure 2 PANNI IgG on ovarian and breast cancer cells viability.

Notes: Reductions in cell viability ( \pm standard error of mean) of A2008, A2780, and SKOV3 human ovarian cancer cells and MCF-7 breast cancer cells compared to vehicle controls produced by incubation for 24 hours with increasing concentrations of PANNI antibodies as an IgG preparation ( $\mathrm{n}=6$ at each concentration). These antibodies had no effect on HEK293 cells (not shown). Concentrations are given as fractional dilutions from original antiserum. 


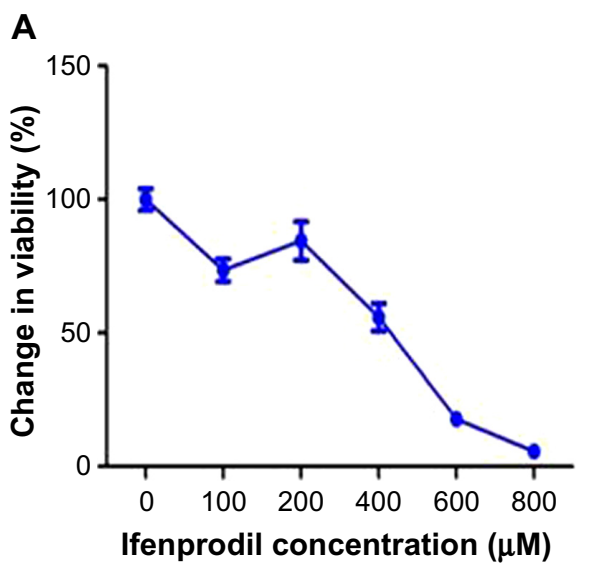

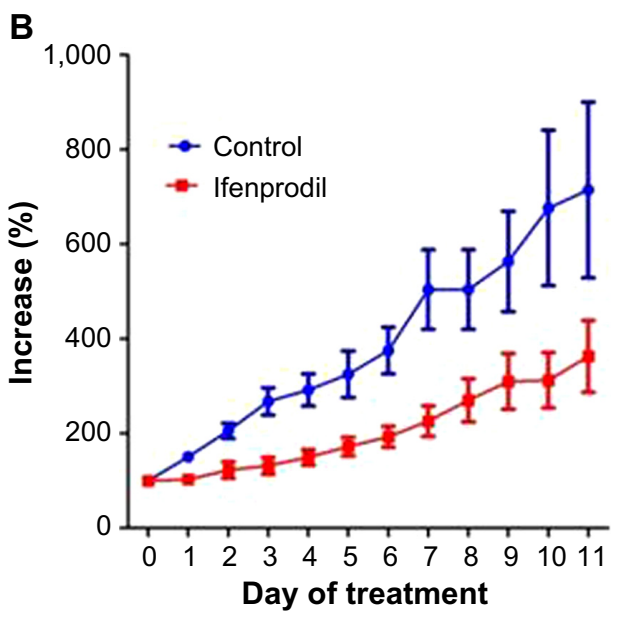

Figure 3 Effect ifenprodil on A2780 cells and tumors.

Notes: (A) Effects ( \pm standard error of mean) on cell viability of A2780 ovarian cancer cells due to incubation with increasing concentrations of GluN2B antagonist ifenprodil compared with vehicle controls over 48 hours. The half-maximal inhibitory concentration was $400 \mu \mathrm{M}(\mathrm{n}=\mathrm{I} 6$ at each concentration). (B) GluN2B antagonist influence on the growth of human ovarian tumor xenografts in nu/nu mice. Administration of ifenprodil at a daily dose of $2.5 \mathrm{mg} / \mathrm{kg}$ body weight for 10 days (days $0-9$ ) significantly reduced growth of $A 2780$ xenografts $(P<0.0 \mathrm{I}, \mathrm{n}=6)$, while having no apparent impact on animal health.

vehicle grew at rapid rates to greater than sevenfold after 8 days of measurement. The GluN2B-specific antagonist ifenprodil, at daily doses of only $2.5 \mathrm{mg} / \mathrm{kg}$ body weight, restricted the growth rate of tumors to threefold over the same period (Figure 3B, $P<0.01$ ). The figure represents the means and standard errors of tumors grown in all six control and all six test animals. However, the tumor in one control animal stopped growing over the course of the treatment period, and the tumor of one test animal seemed to grow at the same rate as tumors from the other control animals and therefore seemed to be unresponsive to ifenprodil. If these two animals were not included in the final data, the influence of the drug on inhibiting growth would have been substantially greater. The body weight and behavior of animals treated with vehicle and ifenprodil showed no differences over and beyond this same period.

\section{Discussion}

The data shown in this paper demonstrate that epithelial ovarian cancer tissue samples and ovarian cancer cell lines express NMDARs, and that these receptors are comprised, at least in part, of GluN1 and GluN2B proteins. Not only is this receptor expressed in these cells but it is also present in the membrane, thereby creating one or more potential targets for cancer treatment. The immunohistochemistry results were from serous papillary cystadenocarcinoma, endometrioid adenocarcinoma, and clear-cell carcinoma, which account for approximately $26 \%, 10 \%$, and $4 \%$ occurrence of ovarian tumors, respectively. All of these tissues showed a positive staining pattern for NMDAR proteins with varying intensities.
There was no distinct correlation between staining intensity and ovarian cancer diagnoses. These immunohistochemistry data suggest that most if not all ovarian cancers will express GluN1 and GluN2B protein, which in turn means these prospective targets could be used as universal treatments. We were unable to detect GluN1and GluN2B expression within normal ovarian tissue, and this suggests the proteins are specific for ovarian cancer and could be potentially used not only for treatment but also diagnosis of ovarian cancer. We were able to verify that the ovarian cancer cell lines also expressed GluN1 protein within the membrane of the cells. These cells were then utilized to demonstrate the functionality of the NMDARs expressed. Targeting these receptors with the PANN1 antibody resulted in significant loss of cell viability. If this finding is reflective of what will happen in vivo, then native anti-GluN1 antibodies, without modification with cytotoxic agents, might destroy ovarian tumors in patients. Preliminary data indicate that the antibody mechanism of action on the ovarian cancer cells possibly involves internalization of the receptors. These data call for future studies to determine if such a change in receptor localization affects key molecules involved in apoptosis, cytotoxicity, and/or cell-cycle control. As could be predicted, we were also able to demonstrate that blocking tumor NMDARs with the small-molecule GluN1 antagonist MK-801 also produced a decrease in cell viability. While all of these findings support the potential use of anti-GluN1 therapeutics, concerns could arise from reports of anti-GluN1 encephalitis occurring in patients through centrally present anti-GluN1 antibodies and immune inflammation, as found 
in responses to ovarian teratomas, small-cell lung cancer, breast cancer, or to autoimmunity of unknown etiology.9-11 While several of these cases are documented, only one of these was in a patient with small-cell lung cancer, one in a patient with breast cancer, and most in patients with ovarian teratoma of germinal cell origin. However, we have previously shown that all small-cell lung cancers and all breast cancers express functional NMDARs, ${ }^{7,8}$ as do apparently all ovarian cancers (from our IHC studies), and only approximately $0.5 \%$ of ovarian cancers are represented by teratomas of germ-cell origin. Therefore, the formation of inflammatory deposits and central anti-GluN1 antibodies seems to involve factors in addition to the expression of NMDARs as surface markers on most tumor types. Tachibana et al proposed that the presence of NMDARs in the cytoplasm of normal ovaries (particularly GluN2B) could explain why anti-GluN1 encephalitis can arise in females of reproductive age who do not have teratomas. ${ }^{12,13}$ However, an absence from most patients with receptor-bearing tumors makes this unlikely. Additionally, support for the likelihood that anti-GluN1 antibody treatments will not adversely affect patients is suggested by the work of During et al, who were able to perform safe oral vaccination in animals against GluN1 receptors. ${ }^{14}$ This appeared to have a protective rather than deleterious effect on brain function. Nevertheless, caution would dictate preferred initial use of Fab fragments of GluN1 antibodies as therapy to prevent possible consequences of central inflammation/invasion.

GluN2B could provide an even safer and more effective therapeutic target in treatment of ovarian cancer. These proteins were shown here as likely consistent components of NMDARs present on the surface of ovarian cancer cells. Moreover, both in vitro and in vivo studies with the GluN2Bspecific inhibitor ifenprodil indicate that anti-GluN2B or Fab fragments of such antibodies would effectively retard or block ovarian cancer growth. This is because ifenprodil produced large reductions in the viability of A2780 cells that were highly significant $(P<0.01)$, with a $50 \%$ reduction at $\sim 400 \mu \mathrm{M}$. Proof of principle was then demonstrated by ifenprodil causing a two- to threefold reduction in ovarian tumor growth in nu/nu mice $(P<0.01)$ at a daily dose of this drug that is approximately 40 times less than the median lethal dose in rats and mice given in Oko Yakuri Pharmacometrics. ${ }^{15}$ No side effects of treatment were apparent, and the weights of treated animals matched those of controls over the course of the study. Since NMDARs in the adult brain comprise chiefly GluN2A, ifenprodil does not cause seizures as small GluN1 antagonists such as MK-801 do, but rather has a neuroprotective effect on brain function. ${ }^{16-20}$ Ifenprodil, which acts at the polyaminebinding site of the GluN2B protein, conceivably itself could prove to be an effective therapeutic agent in the treatment of ovarian cancer. ${ }^{21-23}$ This compound has already been approved for other uses in Japan and Europe. It has also been suggested by others that this antagonist might be an effective drug to improve Parkinson's ataxia and as an analgesic for chronic pain. ${ }^{16-19,24,25}$ While in this study we did not examine for the presence of GluN2A or other subtypes, it has been previously shown these are expressed by small-cell lung cancer and breast cancer. ${ }^{7,8}$ Therefore, there is good reason to believe these subtypes are also expressed by ovarian cancer, but no evidence they impact the capacity of inhibitors, such as ifenprodil, to impair tumor growth.

\section{Author contributions}

WGN drafted the manuscript and was the chief architect of the research conducted. BA and FL were involved in performing confocal, cell-culture, RT-PCR, and binding studies. RY actively participated in performing and planning animal studies, and HA, BA, and FL were jointly responsible for in vitro viability studies. All authors contributed toward drafting and revising the paper and agree to be accountable for all aspects of the work.

\section{Acknowledgments}

We are indebted to Nathan Sylvain, Chenoa Allen, AJ Vincelli, Aki Bowers, Hae-Lin Cho, and Cindy Sun for their technical support. This work was supported in part by PHS grants 19613 and DK07508 to WGN.

\section{Disclosure}

Dartmouth College has taken out a patent on subject matter that is discussed in this article. The authors report no conflicts of interest in this work.

\section{References}

1. Bonfoco E, Krainc D, Ankarcrona M, Nicotera P, Lipton SA. Apoptosis and necrosis: two distinct events induced, respectively, by mild and intense insults with N-methyl-D-aspartate or nitric oxide/superoxide in cortical cell cultures. Proc Natl Acad Sci U S A. 1995;92:7162-7166.

2. Bormann J. Memantine is a potent blocker of N-methyl-D-aspartate (NMDA) receptor channels. Eur J Pharmacol. 1989;166:591-592.

3. Rainer M, Wuschitz A, Jagsch C, Erb C, Chirikdjian JJ, Mucke HA. Memantine in moderate to severe Alzheimer's disease: an observational post-marketing study. J Neural Transm. 2011;2118:1255-1259.

4. Berman FW, Murray TF. Characterization of [3H]MK-801 binding to $\mathrm{N}$-methyl-D-aspartate receptors in cultured rat cerebellar granule neurons and involvement in glutamate-mediated toxicity. $J$ Biochem Toxicol. 1996;11:217-226.

5. Moriyoshi K, Masu M, Ishii T, Shigemoto R, Mizuno N, Nakanishi S. Molecular cloning and characterization of the rat NMDA receptor. Nature. 1991;354:31-37. 
6. Ishii T, Moriyoshi K, Sugihara H, et al. Molecular characterization of the family of the N-methyl-D-aspartate receptor subunits. J Biol Chem. 1993;268:2836-2843.

7. North WG, Gao G, Memoli VA, Pang RH, Lynch L. Breast cancer expresses functional NMDA receptors. Breast Cancer Res Treat. 2010;122:307-314.

8. North WG, Gao G, Jensen A, Memoli V, Du J. NMDA receptors are expressed by small-cell lung cancer and are potential targets for effective treatment. Clin Pharmacol. 2010;2:31-40.

9. Tuzin E, Zhou L, Baehring JM, Bannikh S, Resenfeld MR, Dalmau J. Evidence for antibody-mediated pathogenesis in anti-NMDAR encephalitis associated with ovarian teratoma. Acta Neuropathol. 2009;118: 737-743.

10. Dalmau J, Gleichman AJ, Hughes EG, et al. Anti-NMDA encephalitis: case series and analysis of the effects of antibodies. Lancet Neurol. 2008; 7:1091-1098.

11. Will A, Akalin M. Paraneoplastic limbic encephalitis with NMDA receptor (NR1) antibodies in breast cancer. Neurology. 2012;78:S08.007.

12. Tachibana N, Shirakawa T, Ishii K, et al. Expression of various glutamate receptors including N-methyl-D-aspartate receptor (NMDAR) in ovarian teratoma removed from a young woman with anti-NMDAR encephalitis. Intern Med. 2010;49:2167-2173.

13. Tachibana N, Ikeda S. Localization of NMDAR-related epitopes in ovarian teratoma: comparison between patients and controls. Rinsho Shinkeigaku. 2012;52:982-984.

14. During MJ, Symes CW, Lawlor PA, et al. An oral vaccine against NMDAR1 with efficacy in experimental stroke and epilepsy. Science. 2000;287:1453-1460.

15. Kawai Y, Hirakawa T, Sugisawa A, Motomura I. Acute and subacute toxicity studies of ifenprodil. Oyo Yakuri Pharmacometrics. 1975;10:785

16. Shalaby IA, Chenard BL, Prochniak MA, Butler TW. Neuroprotective effects of the N-methyl-D-aspartate receptor antagonists ifenprodil and SL-82.0715 on hippocampal cells in culture. J Pharmacol Exp Ther. 1992;260:925-932.
17. Graham D, Darles G, Langer SZ. The neuroprotective properties of ifenprodil, a novel NMDA receptor antagonist, in neuronal cell culture toxicity studies. Eur J Pharmacol. 1992;226:373-376.

18. Mishra V, Verma R, Singh N, Raghubir R. The neuroprotective effects of NMDAR antagonist, ifenprodil and ASIC1a inhibitor, flurbiprofen on post-ischemic cerebral injury. Brain Res. 2011;1389: $152-160$.

19. Zhang W, Shi CX, Gu XP, Ma ZL, Zhu W. Antinociception and decreased the expression of NR2B subunits in the dorsal horn after chronic dorsal root ganglia compression in rats. Anesth Analg. 2009;108: 1015-1020.

20. Reynolds IJ, Miller RJ. Ifenprodil is a novel type of N-methylD-aspartate receptor antagonist: interaction with polyamines. Mol Pharmacol. 1989;36:758-765.

21. Williams K. Ifenprodil discriminates subtypes of N-methyl-aspartate receptor: selectivity and mechanisms at recombinant heteromeric receptors. Mol Pharmacol. 1993;44:851-859.

22. Gallagher MJ, Huang H, Pritchett DB, Lynch DR. Interactions between ifenprodil and the NR2B subunit of the N-methyl-D-aspartate receptor. J Biol Chem. 1996;271:9603-9611.

23. Bernardi M, Bertolini A, Szczawinska K, Genedani S. Blockage of polyamine site of NMDA receptors produces antinociception and enhances the effect of morphine in mice. Eur J Pharmacol. 1996;298: 51-55.

24. Chizh BA, Headley PM, Tzschentke TM. NMDA receptor antagonists as analgesics focus on the NR2B subtype. Trends Pharmacol Sci. 2001;22:636-642.

25. Patent Abstracts of Japan Vol 10 no.377 (c-392) 16 December, 1986; and JP-A-61 72820(Grelan Pharmaceutical Co, Ltd), 4 December, 1986.
Clinical Pharmacology: Advances and Applications

\section{Publish your work in this journal}

Clinical Pharmacology: Advances and Applications is an international, peer-reviewed, open access journal publishing original research, reports, reviews and commentaries on all areas of drug experience in humans. The manuscript management system is completely online and includes a very quick and fair peer-review system, which is all easy to use.

\section{Dovepress}

Visit http://www.dovepress.com/testimonials.php to read real quotes from published authors. 